\title{
Establishment of a Comprehensive Epilepsy Center in Pakistan: Initial Experiences, Results, and Reflections
}

\author{
M. Zubair Tahir, ${ }^{1}$ Zain A. Sobani, ${ }^{1}$ S. A. Quadri, ${ }^{1}$ S. Nizam Ahmed, ${ }^{2}$ Mughis Sheerani, ${ }^{3}$ \\ Fowzia Siddiqui, ${ }^{3}$ Warren W. Boling, ${ }^{4}$ and Syed Ather Enam ${ }^{1}$
}

${ }^{1}$ Section of Neurosurgery, Department of Surgery, Aga Khan University, Stadium Road, Karachi, Pakistan

${ }^{2}$ Department of Neurology, University of Alberta Hospital Edmonton, AB, Canada

${ }^{3}$ Section of Neurology, Department of Medicine, Aga Khan University, Stadium Road, Karachi, Pakistan

${ }^{4}$ Department of Neurosurgery, University of Melbourne Parkville, VIC, Australia

Correspondence should be addressed to Syed Ather Enam, ather.enam@aku.edu

Received 16 May 2011; Revised 22 October 2011; Accepted 20 November 2011

Academic Editor: Seyed M. Mirsattari

Copyright ( $) 2012$ M. Zubair Tahir et al. This is an open access article distributed under the Creative Commons Attribution License, which permits unrestricted use, distribution, and reproduction in any medium, provided the original work is properly cited.

\begin{abstract}
Background. Developing countries, home to $80 \%$ of epilepsy patients, do not have comprehensive epilepsy surgery programs. Considering these needs we set up first epilepsy surgery center in Pakistan. Methods. Seventeen teleconferences focused on setting up an epilepsy center at the Aga Khan University (AKU), Karachi, Pakistan were arranged with experts from the University of Alberta Hospital, Alberta, Canada and the University of West Virginia, USA over a two-year period. Subsequently, the experts visited the proposed center to provide hands on training. During this period several interactive teaching sessions, a nationwide workshop, and various public awareness events were organized. Results. Sixteen patients underwent surgery, functional hemispherectomy (HS) was done in six, anterior temporal lobectomy (ATL) in six, and neuronavigation-guided selective amygdalohippocampectomy ( $\mathrm{SAH}$ ) using keyhole technique in four patients. Minimal morbidity was observed in ATL and, SAH groups. All patients in SAH group (100\%) had Grade 1 control, while only 5 patients (83\%) in ATL group, and 4 patients (66\%) in HS group had Grade 1 control according to Engel's classification, in average followups of 12 months, 24 months and 48 months for SAH, ATL, and HS, respectively. Conclusion. As we share our experience we hope to set a practical example for economically constrained countries that successful epilepsy surgery centers can be managed with limited resources.
\end{abstract}

\section{Introduction}

A patient with epilepsy, whose seizures cannot be controlled after at least two years having tried and failed two major antiepileptic drugs (AEDs) with demonstrated therapeutic levels, is regarded as medically refractory [1-3]. Approximately $35 \%$ of patients with epilepsy are refractory to medical therapy [4]. In such cases where medical management has failed, surgery is generally considered as the next step in the management of their ailment. Surgery is indicated in lesionrelated symptomatic epilepsy syndromes such as temporal lobe epilepsy (TLE) with mesial temporal sclerosis (MTS), intracranial tumors, and cortical dysplasias. The number of medically refractory patients as well as lesion-related epilepsy patients is higher in the developing countries due to the higher incidence of infections and perinatal asphyxia leading to greater cortical pathologies. Regrettably, many of the developing countries do not have comprehensive epilepsy centers, leaving patients to suffer with incapacitating seizures and other lifelong comorbidities.

In 1997 the World Health Organization, the International League Against Epilepsy, and the International Bureau for Epilepsy launched a Global Campaign against Epilepsy "Out of the Shadows," from Geneva as well as Dublin, during the 22nd International Congress of Epilepsy. The campaign aimed to increase public and professional awareness of epilepsy as a universal and treatable brain disorder, promote public and professional education about epilepsy, identify the needs of people with epilepsy at the national and regional levels, and encourage governments and departments of health 
to address the needs of people with epilepsy including awareness, education, diagnosis, treatment, care, services, and prevention.

However, the position in developing countries did not change significantly. There is a limited literature available on epilepsy surgery and its application in the developing countries, and proposals to possibly set up comprehensive epilepsy center in low income countries have been put forward $[1,5,6]$, and few pragmatic applications have ensued from these proposals. A pilot project to develop a comprehensive epilepsy center in Uganda demonstrated a framework for such a program to function in a very low resource setting [7].

Here we would like to share our experience right from the initial stages of planning, recruitment, arrangement of transnational teleconferences and identification of potential patients to carrying out successful surgeries and training of future human resources. In doing so we hope to set a practical example for economically constrained countries and to make a difference in the quality of life of patients with medically refractory epilepsy.

\section{Importance of International Collaboration in the Developing World}

Of the 50 million people suffering from epilepsy all over the world, $80 \%$ reside in the developing countries, where the estimated incidence is as high as 190 per $100,000[8,9]$. Approximately $90 \%$ of these patients are deprived of proper treatment due to a lack of resources and proper medical facilities, leading to social stigma, isolation and discrimination $[10,11]$. Further they are subjected to unemployment, inadequate access to education, and psychiatric comorbidities [1216]. All these put together result in severe depression; a study conducted in a hospital setting with focused clinic psychiatric evaluation found that nearly $60 \%$ of patients with epilepsy suffered from depression at the time of interview [17].

International collaboration between a developed and developing countries in the treatment of patients with epilepsy is mutually beneficial [18]. Both collaborating partners develop joint strategies to medically and surgically treat patients within restricted resources. If the center in the developed country has experience in a certain area such as epilepsy surgery, that experience will also help to gain the confidence of the patients in the developing countries. The Canadian Neurological Sciences Federation (CNSF) and Canadian Society of Clinical Neurophysiologists (CSCN) are developing means to improve communications between the Canadian physicians and those in the developing countries (Personal communication with Seyed M. Mirsattari, president CSCN).

We need not look far in the developing world to see such examples, South Africa, a country of 38 million people, has only 8000 registered medical practitioners out of which only 18 are neurologists, [5] and there is just one state hospital carrying out epilepsy surgery [19]. In 1997 there were no trained neurologists in Namibia, with a complete lack of investigational tools [14]. Nepal, a country of 21 million people, has a workforce of 7 neurologists equipped with 3 magnetic resonance imaging (MRI) scanners, 4 electroencephalograms (EEG) machines, and just 10 computed tomography (CT) scanners [20]. The condition in other developing countries is similar. Few countries like Pakistan, India, and Iran that have trained neurologists and neurosurgeons lack technical resources [5].

Keeping the described scenario in mind, International collaborations among developed and developing countries for setting up comprehensive epilepsy surgery programs are very important. It may not be possible to provide the most sophisticated or costly of investigative technologies in developing countries. However, a model for a comprehensive epilepsy program in the developing world has been developed for treatment of pharmaco-resistant TLE using technology and expertise reasonably available in the developing world [7]. This model relies on partnering with epilepsy experts so that expertise and available knowledge can be shared with the developing countries. In developing countries that have capable neurologists and neurosurgeons, this interaction greatly expands the capacity in developing countries to embark on more expertise driven medical approaches such as the evaluation of pharmaco-resistant epilepsy and treatment with surgery for epilepsy.

\section{Epidemiology of Epilepsy in Pakistan}

The most recent data on the prevalence of epilepsy in Pakistan comes from a population-based survey conducted in 1994. The authors found an overall prevalence rate of 999 in 100,000 , with an increased prevalence in rural areas (1480 in 100,000$)$ as compared to urban areas (740 in 100,000). The authors noted that treatment status was deplorable in rural areas, with only $2 \%$ of patients receiving AEDs in rural settings compared to an equally poor $27 \%$ patients in urban populations [21]. Based on this study, an estimated 1.38 million people suffer from epilepsy in Pakistan. In 1994, the population of Pakistan was estimated to be 126 million, making it the ninth most populous country in the world. The number of these patients eligible for epilepsy surgery remains unclear; however, figures extrapolated from available data suggest that around 34,000-45,000 potential surgical candidates are present in the Pakistani population indicating a dire need for a comprehensive epilepsy center [1].

\section{Steps in Setting up an Epilepsy Surgery Center}

4.1. Choice of Location. The resources required in setting up a comprehensive epilepsy center are substantial; the choice of location is thus of utmost importance. The location must be central and accessible to most parts of the country and nestled close to tertiary medical and diagnostic facilities vital to its operation. Of equal importance is the access via road, rail, and air. Preferably, the comprehensive epilepsy center should be a part of a tertiary medical center in order to better manage presurgical patients and their postoperative care.

In our setting for Pakistan, Karachi appears as an ideal choice. The city ranks as a beta world city, for its significant impact on the global economy. It is home to 18 million 
approximately $11 \%$ of Pakistan's population. Augmenting this resident population is the constant influx of seasonal migrants and traders, providing a wide mix of ethnic and cultural backgrounds. As the major center of trade, commerce, and industry, it has access to all parts of the country via strategic sea, road, rail, and air links.

On the biomedical front Karachi has been a major center for research in the country and the region. It is home to nearly 30 public, 80 registered private hospitals and specialized health units, and 12 recognized medical colleges. Additionally a vast number of health care services are available across the city, owing to which it is a major destination for medical tourism with visitors from across the country and its neighbors.

The Aga Khan University Hospital, which is a private university hospital, pioneering this project, is among the leading hospitals in South Asia, chartered in 1983 and accredited by the Joint Commission International Accreditation (JCIA) in 2006. It is associated with the Aga Khan University, a private, autonomous, and international university that promotes human welfare through research, teaching, and community service.

4.2. Collaborations and Telemedicine. Telemedicine has opened new frontiers for world-wide healthcare and global collaborations. Its applications and implementations as a medical tool have expanded by leaps and bounds over the last decade, providing ease of communication between collaborating centers.

Between June 2006 and July 2008, 17 teleconferences were successfully arranged between the Aga Khan University (AKU), Karachi, Pakistan, and University of Alberta Hospital (UAH), Alberta, Canada [18]. The teleconferences were focused on discussing challenging cases in epilepsy, and brainstorming the setting up of an epilepsy surgery center at AKU under guidance of the previously established center in UAH. In January 2008 the program was extended to include University of West Virginia (UWV) in the collaborations. Dr. Warren W. Boling from UWV visited our center and performed SAH on selected patients along with local neurosurgeons.

File transfer protocols (FTPs) were also successfully established enabling the participants to share power-point presentations, patient details, scans, and video-EEG files effectively. Facets requiring special attention when initially setting up international telemedicine collaboration included the difference of time zones, compatible high-speed connections, audio backup, hardware and software compatibility, and connection security. These important technical and practical aspects need to be kept under consideration along with the possibility of technical incompatibility and failure. Details of our personal experience in successfully establishing telemedicine conferences have been discussed previously in order to aid other developing countries planning to embrace this technology and setup similar collaborations [18].

4.3. Gaining Patient Confidence. Among other factors, lack of knowledge, social stigma, cultural myths, and minimum resources create an impediment for the treatment of epilepsy patients in developing countries. Furthermore if they do manage to cross this barrier, the lack of facilities and trained personnel adds to the shaky confidence these patients have in the surgical management of these problems.

An integral part of establishing a comprehensive epilepsy surgery program is gaining patient confidence in the procedure. After identifying potential candidates for surgery, patients and their families were initially included in the teleconferences to discuss the potential risks and benefits of epilepsy surgery with our collaborators. Multiple interactive sessions were held between the families and the collaborators to remove any doubts before the candidates for surgery were finalized. Interaction with these foreign teams played a central role in gaining confidence in the patients for surgery.

When our collaborators came to the center, numerous public education programs were held for patients and their families.

4.4. Procedures Performed. Patients were carefully selected by our team of epileptologists. For the initial stages in the development of comprehensive epilepsy center, we considered patients who were suffering from medically refractory seizures secondary to MTS or evident brain pathology. After detailed selection and preoperative assessment, 16 patients were operated; of the 16 patients 4 underwent selective amygdalohippocampectomy (SAH) under international collaboration.

The use of cerebral hemispherectomy for seizure control was carried out in patients in whom the seizure focus was lateralized, though diffusely localized to one hemisphere. Major indications for HS included progressive chronic encephalitis (Rasmussen's), extensive Sturge-Weber syndrome, and infantile spasms. Patients who were suffering from TLE secondary to MTS were selected for ATL. Later on there was a change in surgical philosophy by switching to SAH in patients with classic MTS with international collaboration. This transition was smooth as basic requirements like Video EEG and MRI scanner, and frameless neuronavigation guidance system was already available.

For SAH candidates, the surgery was carried out with the assistance of an intraoperative neuronavigation system (Medtronic Stealth System). The patients were positioned supine with their heads fixed in a Mayfield clamp and turned to the contralateral side. A minimally invasive approach was performed with a linear preauricular incision, followed by the dissection of the temporalis muscle. A $3 \times 3$ centimeter craniotomy was performed. The dura was incised using a cruciate incision, followed by a standard middle temporal gyrus dissection developed by Niemeyer and popularized by Olivier [22]. The amygdala and hippocampus were observed under microscope and confirmed by neuronavigation. An attempt was made to perform enbloc resection of these structures. Specimens were then sent for histopathological examination.

Postoperatively the patients were kept in high-dependency units for 24 hours, after which they were shifted to regular care units. The average day of discharge was postoperative 
TABLE 1: Dominant seizure types based on clinical assessment for all the 469 patients included in the study.

\begin{tabular}{lccccc}
\hline Simple partial & Complex partial & Primary GTC & Secondary GTC & $\begin{array}{c}\text { Others, for example, } \\
\text { JME, BRE }\end{array}$ & Unclassified \\
\hline $7 \%$ & $16 \%$ & $43 \%$ & $19 \%$ & $3 \%$ & $12 \%$ \\
\hline
\end{tabular}

day 3. After discharge each patient was regularly followed by a neurosurgeon and an epileptologist. Postoperative AED management was under the discretion of the epileptologist.

\section{Results}

Six hundred and nineteen patients either visited or were referred to the epilepsy clinic, out of which 573 records were accessible for review. One hundred and four patient records were excluded from the study as the diagnosis of epilepsy was not established. Four hundred and sixty-nine patients were diagnosed as true cases of epilepsy, confirmed by either of the two epileptologists (Table 1). The visiting patients included $252(53.6 \%)$ males and $217(46.3 \%)$ females. Eight of the patients had history of seizures since birth, and 278 patients $(60 \%)$ had their first seizure before the age of 12 years.

Three hundred and six patients underwent EEG evaluation of which eighty-eight patients' (28.8\% of 316) EEG reports demonstrated focal abnormalities and 66 (22\% of 316) had more specific findings supporting TLE (temporal spikes, sharp waves, or dysfunction). EEGs were not performed in the remaining patients due to the patient's financial situation or the fact that the neurologists were able to clinically classify and manage the patients based on their clinical manifestations and history. The neurologists diagnosed 84 patients with TLE. The average cost of EEG in Pakistan is US\$ 50 (Figure 1).

Seventy four of the 84 patients diagnosed as TLE (88\%) had undergone EEG testing, and 66 had temporal spikes, sharp waves, slow waves, or temporal dysfunction. The other 8 patients had normal EEG findings. MRI was done, and a radiologist's report was available for $52(62 \%)$ of the 84 TLE patients. All MRI images of selected patients were reviewed by epileptologist and neurosurgeon as well. Video EEG was not conducted in most of these patients secondary to a lack of resources and financial constraints.

Twenty-four patients with TLE were refractory to drug therapy, while the remaining was either well controlled with medication or undergoing drug trials. Around $10 \%$ of the patients with TLE had mental retardation or delayed milestones, while $7 \%$ of those with TLE had moderate-tosevere psychosocial problems like depression, severe anxiety, or outbursts.

Out of all patients who were candidates for epilepsy surgery, sixteen patients consented for surgery. We retrospectively reviewed these sixteen cases of epilepsy surgery and assessed their outcome on the basis of Engel's classification for seizure control.

Of the sixteen patients who underwent surgery for medically intractable epilepsy, ten were male. Average age was 22 years. Functional hemispherectomy (HS) was done in six patients who had TLE plus anterior temporal lobectomy

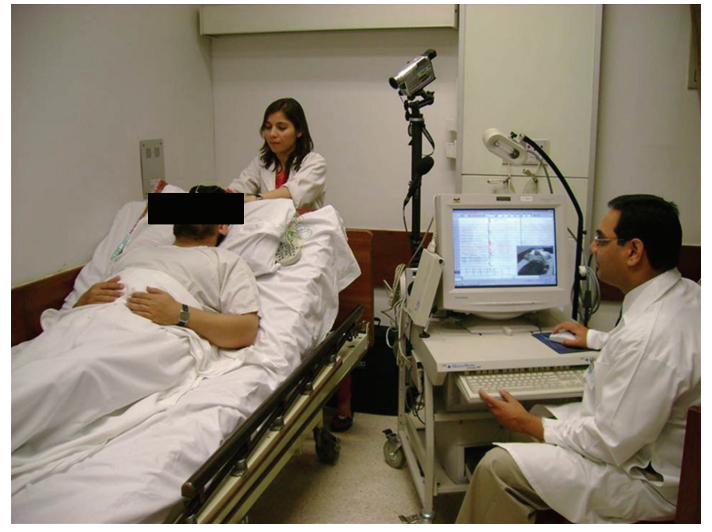

FIGURE 1: One of the epileptologists performing an EEG evaluation on a patient.

(ATL) in six and neuronavigation-guided SAH using keyhole technique in four patients (Figure 2). These four cases of $\mathrm{SAH}$ are those which were carried out after international collaboration. The average operative time for $\mathrm{SAH}$ was two and half hours as compared to three hours in case of ATL. Similarly the average blood loss was $200 \mathrm{cc}$ in SAH as compared to $350 \mathrm{cc}$ in ATL. HS had maximum average blood loss among all the three procedures, which was around $600 \mathrm{cc}$. Because of less invasive technique involved in SAH, patients in this group showed early recovery and shorter hospital stay as compared to other groups. There was minimal morbidity and no mortality in ATL and SAH groups. Poor outcome was encountered in only two patients; both patients had undergone functional HS. One developed hemiplegia and the other died of postoperative acute cerebral edema. The average duration of follow-up was 12 months, 24 months, and 48 months for SAH, ATL, and HS, respectively. Seizure control was assessed using modified Engel's classification. All patients in SAH group (100\%) had Grade 1 control, while 5 patients $(83 \%)$ in ATL group and 4 patients $(66 \%)$ in HS group had Grade 1 control (Table 2).

5.1. Cost. Of the four patients undergoing SAH, two opted for regular inpatient care, while two opted for private care. The average direct cost for the patients undergoing regular inpatient was Rs. 148,000 (US\$ 1644) whereas the direct cost with private care rose significantly to an average of Rs. 274,000 (US\$ 3044). Of interest to note here was that the patients under private care had a longer mean hospital stay (3 days more than those under regular care) due to reluctance of the patient's family to leave early. This cost, however, was the direct cost associated with the procedure and primary admission. It does not include the cost of 


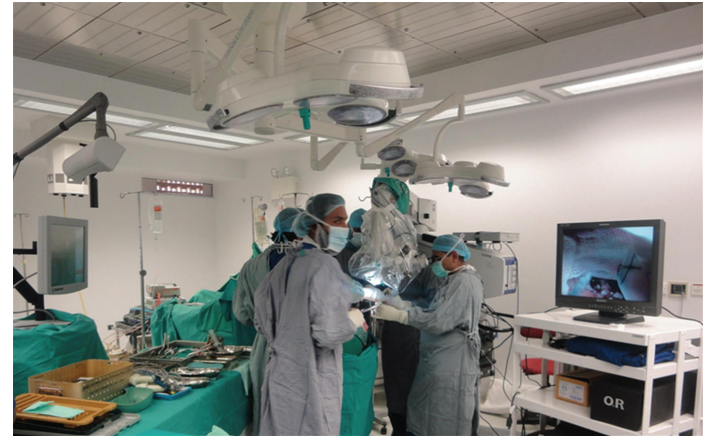

Figure 2: One of our pilot patients undergoing SAH with international collaborators.

TABLE 2: Summary of the procedures performed.

\begin{tabular}{lccc}
\hline & SAH & ATL & HS \\
& $n=4$ & $n=6$ & $n=6$ \\
\hline Blood loss & $200 \mathrm{cc}$ & $350 \mathrm{cc}$ & $600 \mathrm{cc}$ \\
Surgery duration & $2: 30$ hours & 3 hours & 4 hours \\
Hospital stay & 4 days & 8 days & 10 days \\
Followup & 12 months & 24 months & 48 months \\
\hline
\end{tabular}

preoperative evaluation including neurology consultations, MRIs, EEGs, and so forth.

This cost is significantly lower than the cost in other developing countries. In the year 2000, a study came from Chile reporting a total cost of US\$ 5,020 including preoperative evaluation and surgery for an anterior temporal lobectomy (ATL) [23]. Similar paper came from neighboring country India in which the total direct cost of US\$ 5000 was reported for an ATL. Keeping inflation in mind, these costs would be much higher today.

Compared to other centers in the region and other developing countries, our surgical center appears to provide costeffective treatment and in the long run may cater to patients from nearby regions.

5.2. Sustainability of the Program. Even with the collaboration of the international epilepsy surgery team, sustainability of the established program was an important aspect. The whole inpatient and outpatient management was done by residents of the in house neurosurgical program under the supervision of their attending surgeons and the international collaborators. This enabled our team to be actively involved in all aspects of patient care and become acquainted with all stages from preoperative assessment of patients to microsurgical techniques and postoperative care required to carry on epilepsy surgery.

In addition, didactic teaching sessions were conducted, which included seminars and case discussions. Neurosurgeons and residents from across the country were invited to participate in these sessions in order to increase their knowledge in epileptology. Special attention was given to senior residents in order to develop their interest in surgical treatment of epilepsy. Apart from the interactive sessions, numerous formal and informal discussions were arranged with neurologists, neurosurgeons, fellows, technologists, nurses, and medical students interested in epilepsy surgery. A nationwide three-day epilepsy workshop was organized on similar lines as that of the Physician's Assistant Program through Bowman Gray [24].

\section{Conclusion}

As we share our experience in setting up a comprehensive epilepsy center in a developing nation, with international collaboration, we hope to set a practical example for economically constrained countries that comprehensive epilepsy centers can be managed with limited resources.

\section{References}

[1] M. Sheerani, "Development of a comprehensive epilepsy surgery programme in Pakistan," Journal of the Pakistan Medical Association, vol. 55, no. 1, pp. 32-37, 2005.

[2] M. J. Brodie, "Medical therapy of epilepsy: when to initiate treatment and when to combine?" Journal of Neurology, vol. 252, no. 2, pp. 125-130, 2005.

[3] M. J. Brodie and P. Kwan, "Staged approach to epilepsy management," Neurology, vol. 58, no. 8, supplement 5, pp. S2S8, 2002.

[4] P. Kwan and M. J. Brodie, "Early identification of refractory epilepsy," The New England Journal of Medicine, vol. 342, no. 5, pp. 314-319, 2000.

[5] A. A. Asadi-Pooya and M. R. Sperling, "Strategies for surgical treatment of epilepsies in developing countries," Epilepsia, vol. 49, no. 3, pp. 381-385, 2008.

[6] P. N. Sylaja and K. Radhakrishnan, "Problems and pitfalls in developing countries," Epilepsia, vol. 44, no. 1, pp. 48-50, 2003.

[7] W. Boling, A. Palade, A. Wabulya et al., "Surgery for pharmacoresistant epilepsy in the developing world: a pilot study," Epilepsia, vol. 50, no. 5, pp. 1256-1261, 2009.

[8] M. Placencia, J. W. A. S. Sander, M. Roman et al., "The characteristics of epilepsy in a largely untreated population in rural Ecuador," Journal of Neurology Neurosurgery and Psychiatry, vol. 57, no. 3, pp. 320-325, 1994.

[9] A. S. Winkler, M. Schaffert, and E. Schmutzhard, "Epilepsy in resource poor countries-suggestion of an adjusted classification," Epilepsia, vol. 48, no. 5, pp. 1029-1030, 2007.

[10] E. Perucca, "Treatment of epilepsy in developing countries," British Medical Journal, vol. 334, no. 7605, pp. 1175-1176, 2007.

[11] R. A. Scott, S. D. Lhatoo, and J. W. Sander, "The treatment of epilepsy in developing countries: where do we go from here?" Bulletin of the World Health Organization, vol. 79, no. 4, pp. 344-351, 2001.

[12] A. O. Adewuya and B. A. Ola, "Prevalence of and risk factors for anxiety and depressive disorders in Nigerian adolescents with epilepsy," Epilepsy and Behavior, vol. 6, no. 3, pp. 342$347,2005$.

[13] A. O. Adewuya and S. B Oseni, "Impact of psychiatric morbidity on parent-rated quality of life in Nigerian adolescents with epilepsy," Epilepsy and Behavior, vol. 7, no. 3, pp. 497501, 2005. 
[14] P. Jallon, "Epilepsy in developing countries," Epilepsia, vol. 38, no. 10, pp. 1143-1151, 1997.

[15] U. Seneviratne, P. Rajapakse, R. Pathirana, and T. Seetha, "Knowledge, attitude, and practice of epilepsy in rural Sri Lanka," Seizure, vol. 11, no. 1, pp. 40-43, 2002.

[16] R. Tekle-Haimanot, L. Forsgren, A. Gebre-Mariam et al., "Attitudes of rural people in central Ethiopia towards leprosy and a brief comparison with observations on epilepsy," Leprosy Review, vol. 63, no. 2, pp. 157-168, 1992.

[17] A. U. Yousafzai, A. W. Yousafzai, and R. Taj, "Frequency of depression in epilepsy: a hospital based study," Journal of Ayub Medical College, Abbottabad, vol. 21, no. 2, pp. 73-75, 2009.

[18] S. N. Ahmed, C. Mann, F. Siddiqui et al., "Experiences from an international tele-epilepsy collaboration," Canadian Journal of Neurological Sciences, vol. 36, no. 5, pp. 582-586, 2009.

[19] R. Eastman, "Epilepsy in South Africa," Acta Neurologica Scandinavica, vol. 181, pp. 8-11, 2005.

[20] K. C. Rajbhandari, "Epilepsy in Nepal," Canadian Journal of Neurological Sciences, vol. 31, no. 2, pp. 257-260, 2004.

[21] H. Aziz, S. M. Ali, P. Frances, M. I. Khan, and K. Z. Hasan, "Epilepsy in Pakistan: a population-based epidemiologic study," Epilepsia, vol. 35, no. 5, pp. 950-958, 1994.

[22] W. S. Boling, "Selective amygdalohippocampectomy," in Operative Techniques in Epilepsy Surgery, G. Baltuch and J.-G. Villemure, Eds., Thieme Medica, New York, NY, USA, 2009.

[23] M. G. Campos, J. Godoy, M. T. Mesa, G. Torrealba, R. Gejman, and I. Huete, "Temporal lobe epilepsy surgery with limited resources: results and economic considerations," Epilepsia, vol. 41, supplement 4, pp. S18-S21, 2000.

[24] H. T. Wilson, “The present status of the physician's assistant program of the Bowman Gray school of medicine," North Carolina Medical Journal, vol. 35, no. 5, pp. 292-294, 1974. 


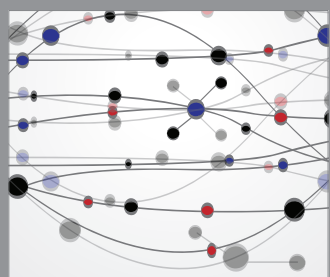

The Scientific World Journal
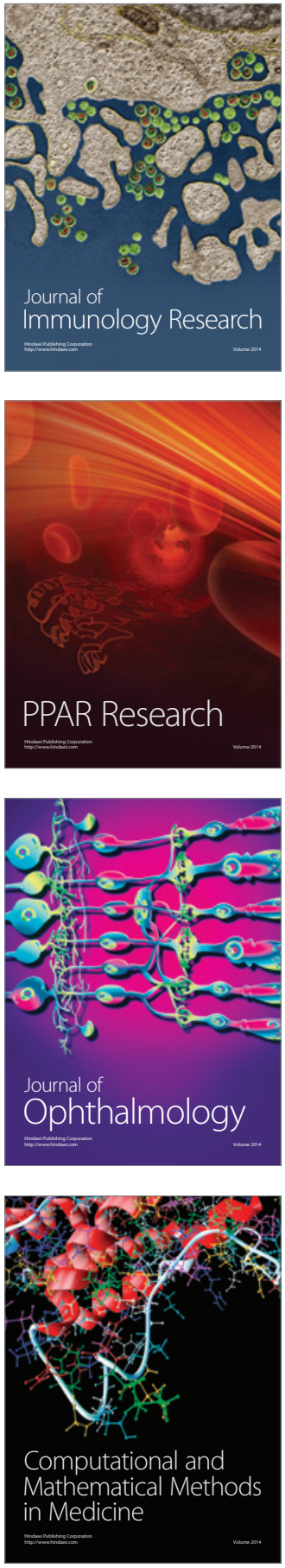

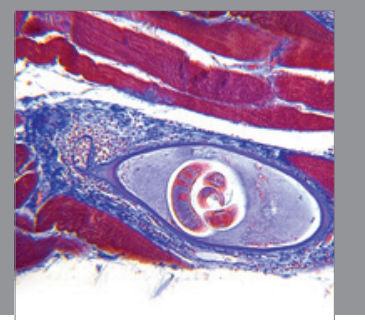

Gastroenterology

Research and Practice
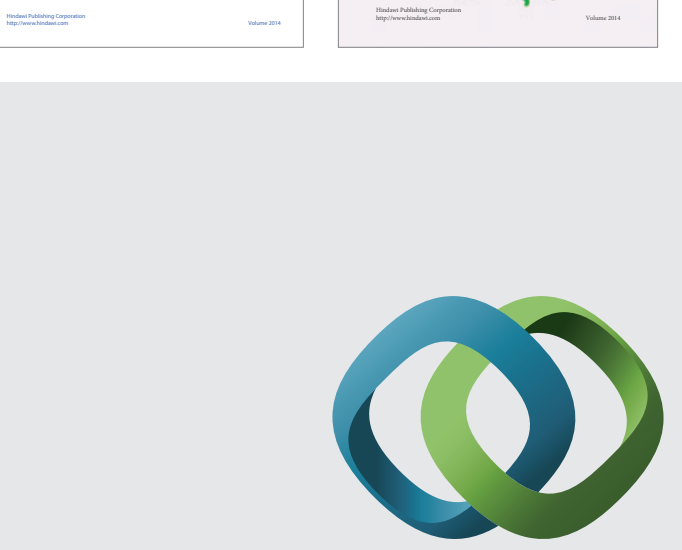

\section{Hindawi}

Submit your manuscripts at

http://www.hindawi.com
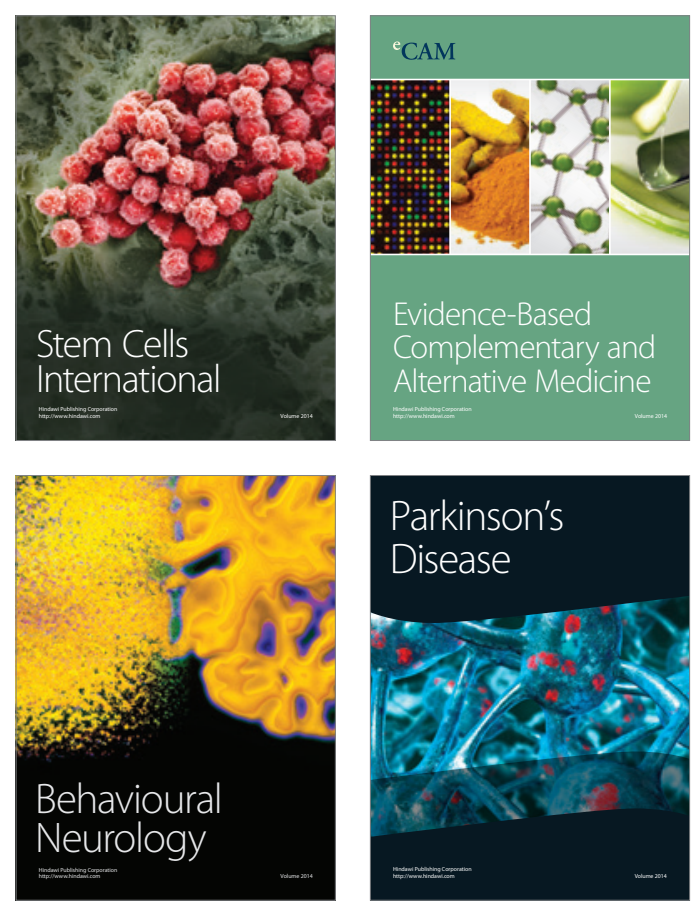

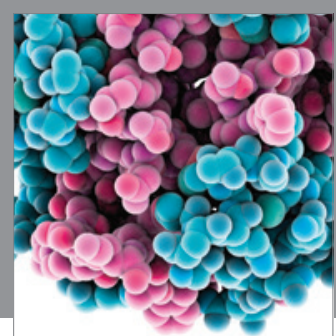

Journal of
Diabetes Research

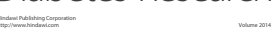

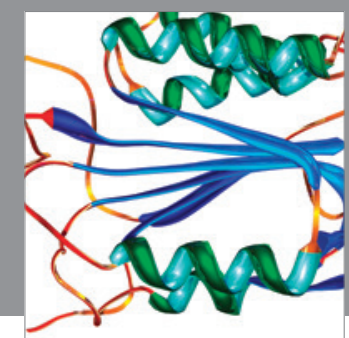

Disease Markers
